\begin{tabular}{|c|l|}
\hline Title & Inequivalence of quantum Dirac fields of different masses and the underlying general structures involved \\
\hline Author(s) & A rai, A sao \\
\hline Citation & $\begin{array}{l}31-53 \\
\text { https://doi.org/10.4171/175-1/2 } \\
\text { Functional analysis and operator theory for quantum physics }\end{array}$ \\
\hline Issue Date & $2017-05$ \\
\hline Doc URL & http://hdl.handle.net/2115/68217 \\
\hline Type & bookchapter (author version) \\
\hline File Information & A rai-Dirac.pdf \\
\hline
\end{tabular}

Instructions for use 


\title{
Inequivalence of Quantum Dirac Fields of Different Masses and the Underlying General Structures Involved
}

\author{
Asao Arai* \\ Department of Mathematics, Hokkaido University \\ Kita 10-Nishi 8, Sapporo, 060-0810, Japan \\ E-mail: arai@math.sci.hokudai.ac.jp
}

Dedicated to Professor Pavel Exner on the occasion of his 70th birthday

\begin{abstract}
A family of irreducible representations of the canonical anticommutation relations over an abstract Hilbert space indexed by a set of bounded linear operators is presented and a theorem on the mutual equivalence of them is established. As an application of the theorem, it is proved that quantum Dirac fields of different masses are mutually inequivalent. Moreover, a new class of irreducible representations of the CAR over a Hilbert space, which includes, as a special case, time-zero quantum Dirac fields, is constructed.
\end{abstract}

2010 MSC: Primary 81-T10; Secondary 47-L55.

Keywords: canonical anticommutation relations, fermion Fock space, inequivalent representation, mass, quantum Dirac field.

\section{Introduction}

In a previous paper [2], the author showed that there exists a general mathematical structure behind the fact [6, Theorem X.46] that the time-zero Hermitian scalar fields of different masses as representations of the canonical commutation relations (CCR) over $L_{\mathbb{R}}^{2}\left(\mathbb{R}^{3}\right)$ (the real Hilbert space of square integrable functions on $\mathbb{R}^{3}$ ) are mutually inequivalent, an interesting fact which may allow one to view the boson masses as objects distinguishing elements in a family of inequivalent representations of the CCR over $L_{\mathbb{R}}^{2}\left(\mathbb{R}^{3}\right)$, giving a

*Supported by JSPS KAKENHI No.15K04888. 
representation theoretic meaning to the boson masses. A point in [2] lies in understanding that the family of time-zero Hermitian scalar fields, which is indexed by the mass parameter, is a special example of a general class of representations of the CCR over an abstract Hilbert space indexed by a set of (unbounded) self-adjoint operators and the inequivalence of the time-zero scalar fields of different masses can be derived as a simple application of a theorem on inequivalence of the representations under consideration in the abstract framework. Based on this structure, a new class of representations of the CCR over $L_{\mathbb{R}}\left(\mathbb{R}^{d}\right)$ with $d \in \mathbb{N}$ arbitrary, including as a special case the time-zero Hermitian scalar fields mentioned above, was found [2].

As a next step of research, it is natural to ask if there exist similar structures in the case of Fermi fields, typically quantum Dirac fields. In this paper, we show that the answer to the question is in the affirmative.

In Section 2, we introduce a family of irreducible representations of the canonical anticommutation relations (CAR) over an abstract Hilbert space $\mathscr{H}$, where their representation space is taken to be the fermion Fock space over $\mathscr{H}$. To the author's best knowledge, this family of representations of CAR may be new. We prove a theorem on inequivalence of these representations. In Section 3, we construct a free quantum Dirac field in the $(1+d)$-dimensional space-time on the fermion Fock space over $L^{2}\left(\mathbb{R}^{d *} ; \mathbb{C}^{\nu}\right)$, the Hilbert space of $\mathbb{C}^{\nu}$-valued square integrable functions on $\mathbb{R}^{d *}$ (the $d$-dimensional wave vector space or the $d$-dimensional momentum space ${ }^{1}$ ), where $\nu$ is defined by (19) below. As an application of the inequivalence theorem in Section 2, we prove that the free quantum Dirac fields of different masses as well as interacting ones are inequivalent. In Section 4, we construct a general class of inequivalent representations of the CAR over $L^{2}\left(\mathbb{R}^{d *} ; \mathbb{C}^{\nu}\right)$, which is a generalization of time-zero quantum Dirac fields. In the last section, we consider quantum Dirac fields on a $d$-dimensional box $M$ for comparison with those on $\mathbb{R}^{d}$. We prove that the quantum Dirac fields on $M$ of different positive masses are equivalent if and only if $d=1$.

\section{A family of irreducible representations of the CAR over a Hilbert space}

The inner product and the norm of a Hilbert space $\mathscr{H}$ are denoted by $\langle\cdot, \cdot\rangle_{\mathscr{H}}$ (antilinear in the left variable) and $\|\cdot\|_{\mathscr{H}}$ respectively. But we sometimes omit the subscript $\mathscr{H}$ in $\langle\cdot, \cdot\rangle_{\mathscr{H}}$ and $\|\cdot\|_{\mathscr{H}}$ if there is no danger of confusion. For a linear operator $A$ on a Hilbert space, we denote its domain by $D(A)$. If $A$ is densely defined, then we denote its adjoint by $A^{*}$.

We first recall the concept of representation of CAR:

\footnotetext{
${ }^{1}$ We use the physical unit system where $\hbar$ (the Planck constant divided by $2 \pi$ ) and the light speed $c$ are equal to 1 .
} 
Definition 2.1 Let $\mathfrak{F}$ and $\mathscr{H}$ be complex Hilbert spaces, and

$$
\mathfrak{A}(\mathscr{H}):=\left\{\psi(f), \psi(f)^{*} \mid f \in \mathscr{H}\right\}
$$

be a subset of $\mathfrak{B}(\mathfrak{F})$, the Banach space of everywhere defined bounded linear operators on $\mathfrak{F}$.

1. The pair $(\mathfrak{F}, \mathfrak{A}(\mathscr{H}))$ is called a representation of the $C A R$ over $\mathscr{H}$ if the following (a) and (b) hold:

(a) Antilineality: For all $f, g \in \mathscr{H}$ and $\alpha, \beta \in \mathbb{C}, \psi(\alpha f+\beta g)=\alpha^{*} \psi(f)+\beta^{*} \psi(g)$.

(b) CAR: For all $f, g \in \mathscr{H}$,

$$
\{\psi(f), \psi(g)\}=0, \quad\left\{\psi(f), \psi(g)^{*}\right\}=\langle f, g\rangle,
$$

where $\{X, Y\}:=X Y+Y X$.

2. The representation $(\mathfrak{F}, \mathfrak{A}(\mathscr{H}))$ is said to be irreducible if there exist no proper subspaces of $\mathfrak{F}$ which remain invariant under the action of all operators $\psi(f)$ and $\psi(f)^{*}$ $(f \in \mathscr{H})$ in $\mathfrak{A}(\mathscr{H})$.

3. Let $\left(\mathfrak{F}^{\prime}, \mathfrak{A}^{\prime}(\mathscr{H})\right)$ with $\left.\mathfrak{A}^{\prime}(\mathscr{H}):=\left\{\psi^{\prime}(f), \psi^{\prime}(f)^{*} \mid f \in \mathscr{H}\right\}\right)$ be another representation of the $C A R$ over $\mathscr{H}$. Then the two representations $\left(\mathfrak{F}^{\prime}, \mathfrak{A}^{\prime}(\mathscr{H})\right)$ and $(\mathfrak{F}, \mathfrak{A}(\mathscr{H}))$ are said to be equivalent if there exists a unitary operator $\mathbb{U}: \mathfrak{F} \rightarrow \mathfrak{F}^{\prime}$ such that, for all $f \in \mathscr{H}, \psi^{\prime}(f)=\mathbb{U} \psi(f) \mathbb{U}^{*}$.

Remark 2.2 Taking the adjoint of the first equation in (b) in Definition 2.1-(1), we have $\left\{\psi(f)^{*}, \psi(g)^{*}\right\}=0, f, g \in \mathscr{H}$.

As is well known, representations of the CAR over a Hilbert space can be constructed on fermion Fock spaces (e.g., [1, Chapter 5], [3, §5.2], [4] and [8]). For the reader's convenience, we first review elementary aspects of fermion Fock spaces.

Let $\mathscr{H}$ be a complex Hilbert space. Then the fermion Fock space $\mathfrak{F}(\mathscr{H})$ over $\mathscr{H}$ is defined as the infinite direct sum Hilbert space

$$
\mathfrak{F}(\mathscr{H}):=\oplus_{n=0}^{\infty} \wedge^{n} \mathscr{H}
$$

of the $n$-fold antisymmetric tensor product Hilbert space $\wedge^{n} \mathscr{H}$ of $\mathscr{H}$ with convention $\wedge^{0} \mathscr{H}:=\mathbb{C}$.

For each $f \in \mathscr{H}$, the creation operator $A^{\dagger}(f)$ with test vector $f$ acting in $\mathfrak{F}(\mathscr{H})$ is defined by

$$
\begin{array}{r}
D\left(A^{\dagger}(f)\right):=\left\{\Psi=\left\{\Psi^{(n)}\right\}_{n=0}^{\infty} \in \mathfrak{F}(\mathscr{H}) \mid \sum_{n=1}^{\infty} n\left\|A_{n}\left(f \otimes \Psi^{(n-1)}\right)\right\|^{2}<\infty\right\}, \\
\left(A^{\dagger}(f) \Psi\right)^{(0)}:=0, \quad\left(A^{\dagger}(f) \Psi\right)^{(n)}:=\sqrt{n} A_{n}\left(f \otimes \Psi^{(n-1)}\right), \quad n \geq 1, \Psi \in D\left(A^{\dagger}(f)\right),
\end{array}
$$


where $A_{n}$ denotes the antisymmetrization operator on the $n$-fold tensor product Hilbert space $\otimes^{n} \mathscr{H}$ of $\mathscr{H}$. Since $D\left(A^{\dagger}(f)\right)$ includes the finite particle subspace

$$
\mathfrak{F}_{0}:=\left\{\Psi \in \mathfrak{F}(\mathscr{H}) \mid \exists n_{0} \text { such that, for all } n \geq n_{0}, \Psi^{(n)}=0\right\},
$$

which is dense in $\mathfrak{F}(\mathscr{H})$, it follows that $D\left(A^{\dagger}(f)\right)$ is dense in $\mathfrak{F}(\mathscr{H})$. Hence the adjoint

$$
A(f):=\left(A^{\dagger}(f)\right)^{*}
$$

of $A^{\dagger}(f)$ exists and is called the annihilation operator with test vector $f$. It follows that $A(f)^{*}=A^{\dagger}(f)$. We denote by $A(f)^{\#}$ either $A(f)$ or $A(f)^{*}$.

It is proved (e.g., [1, Chapter 5] and $[3, \S 5.2])$ that $D\left(A(f)^{\#}\right)=\mathfrak{F}(\mathscr{H})$ and $A(f)^{\#}$ is bounded satisfying the following anticommutation relations:

$$
\{A(f), A(g)\}=0, \quad\left\{A(f), A(g)^{*}\right\}=\langle f, g\rangle, \quad f, g \in \mathscr{H} .
$$

The correspondence: $\mathscr{H} \ni f \mapsto A(f)$ (resp. $\left.A(f)^{*}\right)$ is complex antilinear (resp. linear). Thus $\left(\mathfrak{F}(\mathscr{H}),\left\{A(f), A(f)^{*} \mid f \in \mathscr{H}\right\}\right)$ is a representation of the CAR over $\mathscr{H}$, which is called the Fock representation of the CAR over $\mathscr{H}$. It is well known that it is irreducible (e.g., [8, Theorem 10.2]).

We now fix an orthogonal decomposition

$$
\mathscr{H}=\mathscr{H}_{+} \oplus \mathscr{H}_{-}
$$

of $\mathscr{H}$ with $\mathscr{H}_{+}$and $\mathscr{H}_{-}$being mutually orthogonal nontrivial closed subspaces $\left(\mathscr{H}_{+} \neq\right.$ $\{0\}, \mathscr{H}$ ) and a conjugation $C$ on $\mathscr{H}\left(C\right.$ is an antilinear mapping on $\mathscr{H}$ satisfying $C^{2}=I$ (identity) and $\|C f\|=\|f\|, f \in \mathscr{H})$. We have

$$
\langle C f, C g\rangle=\langle g, f\rangle, \quad f, g \in \mathscr{H} .
$$

We denote by $\mathfrak{B}_{ \pm}$the Banach space of everywhere defined bounded linear operators from $\mathscr{H}$ to $\mathscr{H}_{ \pm}$and introduce a subset of the direct product space $\mathfrak{B}_{+} \times \mathfrak{B}_{-}$:

$$
\mathfrak{T}(\mathscr{H}):=\left\{T=\left(T_{+}, T_{-}\right) \mid T_{ \pm} \in \mathfrak{B}_{ \pm}, T_{+}^{*} T_{+}+\bar{T}_{-}^{*} \bar{T}_{-}=I\right\},
$$

where

$$
\bar{T}_{-}:=C T_{-} C .
$$

It is easy to see that $\bar{T}_{-}$is a bounded linear operator on $\mathscr{H}$ with operator norm $\left\|\bar{T}_{-}\right\|=$ $\left\|T_{-}\right\|$.

Each $T \in \mathfrak{T}(\mathscr{H})$ defines an element of $\mathfrak{B}(\mathscr{H})$ by

$$
T f:=\left(T_{+} f, T_{-} f\right), \quad f \in \mathscr{H} .
$$

For each $T \in \mathfrak{T}(\mathscr{H})$, we define an antilinear mapping $\psi_{T}: \mathscr{H} \rightarrow \mathfrak{B}(\mathfrak{F})$ by

$$
\psi_{T}(f):=A\left(T_{+} f, 0\right)+A\left(0, T_{-} C f\right)^{*}, \quad f \in \mathscr{H} .
$$


It is obvious that

$$
\psi_{T}(f)^{*}=A\left(T_{+} f, 0\right)^{*}+A\left(0, T_{-} C f\right) .
$$

Let

$$
\mathfrak{A}_{T}(\mathscr{H}):=\left\{\psi_{T}(f), \psi_{T}^{*}(f) \mid f \in \mathscr{H}\right\} .
$$

Lemma 2.3 For all $f, g \in \mathscr{H}$, the following anticommutation relations hold:

$$
\begin{array}{r}
\left\{\psi_{T}(f), \psi_{T}(g)\right\}=\left\{\psi_{T}(f)^{*}, \psi_{T}(g)^{*}\right\}=0, \\
\left\{\psi_{T}(f), \psi_{T}(g)^{*}\right\}=\langle f, g\rangle .
\end{array}
$$

Proof. By direct computations using (1), we have

$$
\left\{\psi_{T}(f), \psi_{T}(g)^{*}\right\}=\left\langle T_{+} f, T_{+} g\right\rangle+\left\langle\bar{T}_{-} f, \bar{T}_{-} g\right\rangle .
$$

Since $T_{+}^{*} T_{+}+\bar{T}_{-}^{*} \bar{T}_{-}=I$, we obtain (7). Similarly one can prove (6).

Lemma 2.3 shows that $\left(\mathfrak{F}(\mathscr{H}), \mathfrak{A}_{T}(\mathscr{H})\right)$ is a representation of the CAR over $\mathscr{H}$.

Remark 2.4 The standard choice of $T=\left(T_{+}, T_{-}\right)$in the literature is given by $T_{+}=P$ and $T_{-}=C(I-P) C$, where $P$ is the orthogonal projection onto $\mathscr{H}_{+}$(see, e.g., [4, pp.2223] and [8, §10.1.3]). In this case, the representation is called a quasi-free representation. Hence the representation $\left(\mathfrak{F}(\mathscr{H}), \mathfrak{A}_{T}(\mathscr{H})\right)$ gives a generalization of of the quasi-free representation.

To find more detailed properties of the representation $\left(\mathfrak{F}(\mathscr{H}), \mathfrak{A}_{T}(\mathscr{H})\right)$, we introduce the following additional conditions (T.1)-(T.3) for $T=\left(T_{+}, T_{-}\right) \in \mathfrak{T}(\mathscr{H})$ :

$$
\text { (T.1) } T_{+} T_{+}^{*}=I ;(\mathrm{T} .2) T_{-} T_{-}^{*}=I ;(\mathrm{T} .3) T_{-} \bar{T}_{+}^{*}=0 .
$$

We define a subset of $\mathfrak{T}(\mathscr{H})$ :

$$
\mathfrak{T}_{*}(\mathscr{H}):=\{T \in \mathfrak{T}(\mathscr{H}) \mid(\mathrm{T} .1)-(\mathrm{T} .3) \text { hold }\} .
$$

Lemma 2.5 Let $T \in \mathfrak{T}_{*}(\mathscr{H})$. Then

$$
A(f)=\psi_{T}\left(T_{+}^{*} f_{+}\right)+\psi_{T}\left(C T_{-}^{*} f_{-}\right)^{*}, \quad f=\left(f_{+}, f_{-}\right), f_{ \pm} \in \mathscr{H}_{ \pm} .
$$

Proof. By (T.1) and (T.3), we have

$$
\psi_{T}\left(T_{+}^{*} f_{+}\right)=A\left(f_{+}, 0\right), \quad f_{+} \in \mathscr{H}_{+} .
$$

Property (T.3) implies that

$$
\bar{T}_{+} T_{-}^{*}=0
$$

By this fact and (T.2), we obtain

$$
\psi_{T}\left(C T_{-}^{*} f_{-}\right)=A\left(0, f_{-}\right)^{*}, \quad f_{-} \in \mathscr{H}_{-} .
$$

We have

$$
A(f)=A\left(f_{+}, 0\right)+A\left(0, f_{-}\right) .
$$

Thus we obtain (9). 
Lemma 2.6 For all $T \in \mathfrak{T}_{*}(\mathscr{H}),\left(\mathfrak{F}(\mathscr{H}), \mathfrak{A}_{T}(\mathscr{H})\right)$ is irreducible.

Proof. Let $\mathfrak{D}$ be a closed subspace which is invariant under the action of all $\psi_{T}(f)$ and $\psi_{T}(f)^{*}(f \in \mathscr{H})$. Let $Q$ be the orthogonal projection onto $\mathfrak{D}$. Then it follows that, for all $f \in \mathscr{H}, Q \psi_{T}(f)^{\#}=\psi_{T}(f)^{\#} Q$. Hence, by $(9), Q A(f)^{\#}=A(f)^{\#} Q$ for all $f \in \mathscr{H}$. It is well known (or easy to see) that $\left\{A(f), A(f)^{*} \mid f \in \mathscr{H}\right\}$ is irreducible. Hence $Q=\alpha I$ for some $\alpha \in \mathbb{C}$. But, since $Q$ is an orthogonal projection, it follows that $\alpha=0$ or $\alpha=1$. This means that $\mathfrak{D}=\{0\}$ or $\mathfrak{D}=\mathfrak{F}(\mathscr{H})$. Thus $\mathfrak{A}_{T}(\mathscr{H})$ is irreducible.

Lemmas 2.3 and 2.6 immediately yield the following theorem:

Theorem 2.7 For each $T \in \mathfrak{T}_{*}(\mathscr{H}),\left(\mathfrak{F}(\mathscr{H}), \mathfrak{A}_{T}(\mathscr{H})\right)$ is an irreducible representation of the $C A R$ over $\mathscr{H}$.

Thus we have a family $\left\{\left(\mathfrak{F}(\mathscr{H}), \mathfrak{A}_{T}(\mathscr{H})\right\}_{T \in \mathfrak{T}_{*}(\mathscr{H})}\right.$ of irreducible representations of the CAR over $\mathscr{H}$.

We next consider equivalence or inequivalence of two representations $\left(\mathfrak{F}(\mathscr{H}), \mathfrak{A}_{T}(\mathscr{H})\right)$ and $\left(\mathfrak{F}(\mathscr{H}), \mathfrak{A}_{S}(\mathscr{H})\right)$ with $S \neq T\left(S, T \in \mathfrak{T}_{*}(\mathscr{H})\right)$.

For each pair $(S, T) \in \mathfrak{T}_{*}(\mathscr{H}) \times \mathfrak{T}_{*}(\mathscr{H})$, we define linear operators $V$ and $W$ on $\mathscr{H}$ as follows:

$$
V f:=\left(S_{+} T_{+}^{*} f_{+}, S_{-} T_{-}^{*} f_{-}\right), \quad W f:=\left(S_{+} \bar{T}_{-}^{*} f_{-}, S_{-} \bar{T}_{+}^{*} f_{+}\right), \quad f=\left(f_{+}, f_{-}\right) \in \mathscr{H} .
$$

Lemma 2.8 The following equations hold:

$$
\begin{aligned}
& V^{*} V+\bar{W}^{*} \bar{W}=I, \\
& V V^{*}+W W^{*}=I, \\
& \bar{V}^{*} \bar{W}+W^{*} V=0, \\
& \bar{V} W^{*}+\bar{W} V^{*}=0 .
\end{aligned}
$$

Proof. The operators $V$ and $W$ have the following operator matrix representations:

$$
V=\left(\begin{array}{cc}
S_{+} T_{+}^{*} & 0 \\
0 & S_{-} T_{-}^{*}
\end{array}\right), \quad W=\left(\begin{array}{cc}
0 & S_{+} \bar{T}_{-} * \\
S_{-} \bar{T}_{+}^{*} & 0
\end{array}\right) .
$$

Using these representations and properties of $S$ and $T$, one can easily prove (10)-(13) by direct computations.

We define

$$
B(f):=A(V f)+A(W C f)^{*}, \quad f \in \mathscr{H} .
$$

Then it is easy to see that $\left\{B(f), B(f)^{*} \mid f \in \mathscr{H}\right\}$ is a representation of the CAR over $\mathscr{H}$.

Lemma 2.9 There exists a unitary operator $\mathbb{U}$ on $\mathfrak{F}(\mathscr{H})$ such that

$$
A(f)=\mathbb{U} B(f) \mathbb{U}^{*}, \quad f \in \mathscr{H},
$$

if and only if $W$ is Hilbert-Schmidt. 
Proof. Since we have (10)-(13), the lemma follows from the general theory of Bogoliubov transformations on $\mathfrak{F}(\mathscr{H})$ (see, e.g., $[8, \S 10.3]$ ).

Theorem 2.10 Let $T$ and $S$ be in $\mathfrak{T}_{*}(\mathscr{H})$ with $T \neq S$. Then the two representations $\left(\mathfrak{F}(\mathscr{H}), \mathfrak{A}_{T}(\mathscr{H})\right)$ and $\left(\mathfrak{F}(\mathscr{H}), \mathfrak{A}_{S}(\mathscr{H})\right)$ are equivalent if and only if $S_{+} \bar{T}_{-}^{*}$ and $S_{-} \bar{T}_{+}^{*}$ are Hilbert-Schmidt.

Proof. Suppose that $\left(\mathfrak{F}(\mathscr{H}), \mathfrak{A}_{T}(\mathscr{H})\right)$ and $\left(\mathfrak{F}(\mathscr{H}), \mathfrak{A}_{S}(\mathscr{H})\right)$ are equivalent. Then there exists a unitary operator $\mathbb{U}$ on $\mathfrak{F}(\mathscr{H})$ such that

$$
\mathbb{U} \psi_{S}(f) \mathbb{U}^{*}=\psi_{T}(f), \quad f \in \mathscr{H} .
$$

Then, by (T.1)-(T.3), we have

$$
A\left(f_{+}, 0\right)=\mathbb{U}\left(A\left(S_{+} T_{+}^{*} f_{+}, 0\right)+A\left(0, S_{-} C T_{+}^{*} f_{+}\right)^{*}\right) \mathbb{U}^{*}, \quad f_{+} \in \mathscr{H}_{+} .
$$

and

$$
A\left(0, f_{-}\right)^{*}=\mathbb{U}\left(A\left(S_{+} C T_{-}^{*} f_{-}, 0\right)+A\left(0, S_{-} T_{-}^{*} f_{-}\right)^{*}\right) \mathbb{U}^{*}, \quad f_{-} \in \mathscr{H}_{-} .
$$

Hence, adding the first equation to the second one, we obtain (15). Therefore, by Lemma 2.9, $W$ is Hilbert-Schmidt, which is equivalent to that $S_{+} \bar{T}_{-}^{*}$ and $S_{-} \bar{T}_{+}^{*}$ are HilbertSchmidt.

Conversely, suppose that $S_{+} \bar{T}_{-}^{*}$ and $S_{-} \bar{T}_{+}^{*}$ are Hilbert-Schmidt. Then $W$ is HilbertSchmidt. Hence, by Lemma 2.9 , there exists a unitary operator $\mathbb{U}$ on $\mathfrak{F}(\mathscr{H})$ such that (15) holds. Then (17) and (18) hold. Using the property that $T_{+}^{*} T_{+}+\bar{T}_{-}^{*} \bar{T}_{-}=I$, we obtain (16).

The contraposition of Theorem 2.10 gives an inequivalence theorem on the two representations $\left(\mathfrak{F}(\mathscr{H}), \mathfrak{A}_{T}(\mathscr{H})\right)$ and $\left(\mathfrak{F}(\mathscr{H}), \mathfrak{A}_{S}(\mathscr{H})\right)$ :

Theorem 2.11 Let $T$ and $S$ be in $\mathfrak{T}_{*}(\mathscr{H})$ with $T \neq S$. Then the two representations $\left(\mathfrak{F}(\mathscr{H}), \mathfrak{A}_{T}(\mathscr{H})\right)$ and $\left(\mathfrak{F}(\mathscr{H}), \mathfrak{A}_{S}(\mathscr{H})\right)$ are inequivalent if and only if $S_{+} \bar{T}_{-}^{*}$ or $S_{-} \bar{T}_{+}^{*}$ is not Hilbert-Schmidt.

\section{Inequivalence of quantum Dirac fields of different masses}

In this section, we consider a free quantum Dirac field on the $(1+d)$-dimensional spacetime and an interacting one as well. We take the space dimension $d \in \mathbb{N}$ to be arbitrary, because we want to see the dependence or independence of properties of the Dirac field on $d$. The main purpose of this section is to show by applying Theorem 2.11 that the time-zero quantum Dirac fields of different masses, which are representations of the CAR over a Hilbert space, are mutually inequivalent. This implies that the time- $t$ quantum Dirac fields of different masses $(t \in \mathbb{R})$ also are mutually inequivalent. 


\subsection{The $d$-dimensional free Dirac operator}

For each $d \in \mathbb{N}$, we define $\nu \in \mathbb{N}$ as follows:

$$
\nu:=\left\{\begin{array}{cc}
2^{(d+1) / 2} & \text { if } d \text { is odd } \\
2^{d} & \text { if } d \text { is even }
\end{array} .\right.
$$

It is well known that there exist $\nu \times \nu$ Hermitian matrices $\left\{\alpha_{j}\right\}_{j=1}^{d}$ and $\beta$ satisfying the following anticommutation relations (a representation of the Clifford algebra associated with the Euclidean vector space $\left.\mathbb{R}^{1+d}\right)$ :

$$
\begin{aligned}
& \left\{\alpha_{j}, \alpha_{k}\right\}=2 \delta_{j k} I_{\nu}, \quad j, k=1, \ldots, d, \\
& \left\{\alpha_{j}, \beta\right\}=0, \quad j=1, \ldots, d, \\
& \beta^{2}=I_{\nu},
\end{aligned}
$$

where $\delta_{j k}$ is the Kronecker delta and $I_{n}(n \in \mathbb{N})$ is the $n \times n$ identity matrix.

The free Dirac equation with mass $m \geq 0$ in the $(1+d)$-dimensional space-time $\mathbb{R}^{1+d}:=\left\{(t, x) \mid t \in \mathbb{R}, x=\left(x_{1}, \ldots, x_{d}\right) \in \mathbb{R}^{d}\right\}$ is of the form:

$$
i \frac{\partial \psi(t, x)}{\partial t}=H_{m} \psi(t, x)
$$

where $\psi: \mathbb{R}^{1+d} \rightarrow \mathbb{C}^{\nu}$ (or a $\nu$ component distribution on $\mathbb{R}^{1+d}$ ), $i$ is the imaginary unit and

$$
H_{m}:=\sum_{j=1}^{d} \alpha_{j} p_{j}+m \beta
$$

with $p_{j}:=-i D_{j}\left(D_{j}\right.$ is the generalized partial differential operator in the variable $\left.x_{j}\right)$.

We use the symbol $\mathbb{R}^{d *}=\left\{k=\left(k_{1}, \ldots, k_{d}\right) \mid k_{j} \in \mathbb{R}, j=1, \ldots, d\right\}$ for the dual space of $\mathbb{R}^{d}$ (the space of $d$-dimensional wave vectors) and denote by $\mathscr{F}_{d}: L^{2}\left(\mathbb{R}^{d}\right) \rightarrow L^{2}\left(\mathbb{R}^{d *}\right)$ the $d$-dimensional Fourier transform:

$$
\left(\mathscr{F}_{d} f\right)(k):=\frac{1}{(2 \pi)^{d / 2}} \int_{\mathbb{R}^{d}} f(x) e^{-i k x} d x, \quad f \in L^{2}\left(\mathbb{R}^{d}\right), k \in \mathbb{R}^{d *},
$$

in the $L^{2}$-sense, where $k x:=\sum_{j=1}^{d} k_{j} x_{j}$.

In what follows, we treat $H_{m}$ as an operator acting in

$$
\mathscr{H}_{\mathrm{D}}:=L^{2}\left(\mathbb{R}^{d} ; \mathbb{C}^{\nu}\right)=\left\{f=\left(f_{r}\right)_{r=1}^{\nu} \mid f_{r} \in L^{2}\left(\mathbb{R}^{d}\right), r=1, \ldots, \nu\right\},
$$

the Hilbert space of $\mathbb{C}^{\nu}$-valued square integrable functions on $\mathbb{R}^{d}$. We set

$$
\hat{\mathscr{H}}_{\mathrm{D}}:=\mathscr{F}_{d} \mathscr{H}_{\mathrm{D}}=L^{2}\left(\mathbb{R}^{d *} ; \mathbb{C}^{\nu}\right) \text {. }
$$


For each $k \in \mathbb{R}^{d *}$, we define a $\nu \times \nu$ Hermitian matrix $h_{m}(k)$ by

$$
h_{m}(k):=\alpha k+m \beta,
$$

where $\alpha k:=\sum_{j=1}^{d} \alpha_{j} k_{j}$, and denote the multiplication operator by the matrix-valued function $h_{m}(\cdot)$ on $\hat{\mathscr{H}}_{\mathrm{D}}$ by $\hat{H}_{m}$ :

$$
\begin{aligned}
& D\left(\hat{H}_{m}\right):=\left\{f \in \hat{\mathscr{H}}_{\mathrm{D}} \mid \int_{\mathbb{R}^{d *}}\left\|h_{m}(k) f(k)\right\|_{\mathbb{C}^{\nu}}^{2} d k<\infty\right\}, \\
& \hat{H}_{m} f(k):=h_{m}(k) f(k), \quad f \in D\left(\hat{H}_{m}\right), \text { a.e. } k \in \mathbb{R}^{d *} .
\end{aligned}
$$

By the theory of Fourier transform, we have $\mathscr{F}_{d} p_{j} \mathscr{F}_{d}^{-1}=k_{j}(j=1, \ldots, d)$, where the right hand side denotes the multiplication operator by the variable $k_{j}$. Hence it follows that

$$
\mathscr{F}_{d} H_{m} \mathscr{F}_{d}^{-1}=\hat{H}_{m}
$$

Using (20), one has

$$
(\alpha k)^{2}=k^{2}, \quad k \in \mathbb{R}^{d *} .
$$

By this fact, (21) and (22), one obtains that

$$
\left\|h_{m}(k) f(k)\right\|_{\mathbb{C}^{\nu}}^{2}=\left(k^{2}+m^{2}\right)\|f(k)\|_{\mathbb{C}^{\nu}}^{2}, \quad k \in \mathbb{R}^{d *} .
$$

Hence

$$
D\left(\hat{H}_{m}\right)=\left\{f \in \hat{\mathscr{H}}_{\mathrm{D}} \mid \int_{\mathbb{R}^{d *}} k^{2}\|f(k)\|_{\mathbb{C}^{\nu}}^{2} d k<\infty\right\}
$$

Let

$$
E_{m}(k):=\sqrt{k^{2}+m^{2}}, \quad k \in \mathbb{R}^{d *}
$$

and

$$
\begin{aligned}
& d_{m}(k):=\frac{m+E_{m}(k)+\beta \alpha k}{\sqrt{2 E_{m}(k)\left(m+E_{m}(k)\right)}} \quad(\text { the case } m>0), \\
& d_{0}(k):=\left\{\begin{array}{cc}
\frac{1}{\sqrt{2}}\left(1+\beta \frac{\alpha k}{|k|}\right) & \text { for } k \neq 0 \\
I_{\nu} & \text { for } k=0
\end{array} \quad \text { (the case } m=0\right) .
\end{aligned}
$$

As in the case $d=3$ (e.g., $[8, \S 1.4])$, one can show that $d_{m}(k)$ is unitary and

$$
d_{m}(k) h_{m}(k) d_{m}(k)^{-1}=E_{m}(k) \beta, \quad k \in \mathbb{R}^{d *} .
$$

We denote by $\hat{D}_{m}$ the multiplication operator by $d_{m}(\cdot)$. The operator

$$
U_{m}:=\hat{D}_{m} \mathscr{F}_{d}
$$


is a unitary operator from $\mathscr{H}_{\mathrm{D}}$ to $\hat{\mathscr{H}}_{\mathrm{D}}$. By $(24)$ and $(28)$, we have

$$
U_{m} H_{m} U_{m}^{-1}=E_{m} \beta .
$$

Namely $H_{m}$ is unitarily equivalent to $E_{m} \beta$. It is obvious that $E_{m} \beta$ is self-adjoint. Hence $H_{m}$ is self-adjoint. Thus, for each $\psi_{0} \in D\left(H_{m}\right)$, the free Dirac equation (23) with initial condition $\psi(0, \cdot)=\psi_{0}$ has the unique solution

$$
\psi(t, \cdot)=e^{-i t H_{m}} \psi_{0}, \quad t \in \mathbb{R} .
$$

Relation (29) clarifies spectral properties of $H_{m}$ too. For a linear operator $A$ on a Hilbert space, we denote by $\sigma(A)$ (resp. $\left.\sigma_{\mathrm{p}}(A)\right)$ the spectrum (resp. the point spectrum) of $A$. By the unitary invariance of spectra, (29) implies that

$$
\sigma\left(H_{m}\right)=\sigma\left(E_{m} \beta\right), \quad \sigma_{\mathrm{p}}\left(H_{m}\right)=\sigma_{\mathrm{p}}\left(E_{m} \beta\right) .
$$

It follows from $(22)$ and $\beta \neq \pm I_{\nu}$ that $\sigma(\beta)=\sigma_{\mathrm{p}}(\beta)=\{ \pm 1\}$. Hence $\sigma\left(E_{m} \beta\right)=$ $\left\{E_{m}(k) \mid k \in \mathbb{R}^{d *}\right\} \cup\left\{-E_{m}(k) \mid k \in \mathbb{R}^{d *}\right\}=(-\infty,-m] \cup[m, \infty)$ and $\sigma_{\mathrm{p}}\left(E_{m} \beta\right)=\emptyset$. Therefore

$$
\sigma\left(H_{m}\right)=(-\infty,-m] \cup[m, \infty), \quad \sigma_{\mathrm{p}}\left(H_{m}\right)=\emptyset .
$$

\subsection{Eigenvectors of $h_{m}(k)$ and some operators}

One can easily show that $\operatorname{dim} \operatorname{ker}(\beta \pm 1)=\nu / 2$. Hence, by diagonalization (if necessary), we can assume without loss of generality that

$$
\beta=\left(\begin{array}{cc}
I_{\nu / 2} & 0 \\
0 & -I_{\nu / 2}
\end{array}\right)
$$

We denote by $\left\{e_{r}\right\}_{r=1}^{\nu}$ the standard basis of $\mathbb{C}^{\nu}: e_{r}=\left(\delta_{r r^{\prime}}\right)_{r^{\prime}=1}^{\nu}$. For all $k \in \mathbb{R}^{d *}$ and $s=1, \ldots, \nu / 2$, we define the following vectors in $\mathbb{C}^{\nu}$ :

$$
u_{m}(k, s):=d_{m}(k)^{-1} e_{s} \in \mathbb{C}^{\nu}, \quad v_{m}(k, s):=d_{m}(k)^{-1} e_{s+(\nu / 2)} \in \mathbb{C}^{\nu} .
$$

By (28), we have

$$
h_{m}(k) u_{m}(k, s)=E_{m}(k) u_{m}(k, s), \quad h_{m}(k) v_{m}(k, s)=-E_{m}(k) v_{m}(k, s) .
$$

Namely $u_{m}(k, s)$ (resp. $\left.v_{m}(k, s)\right)$ is an eigenvector of $h_{m}(k)$ with positive (resp. negative) energy $E_{m}(k)$ (resp. $\left.-E_{m}(k)\right)$. Since $d_{m}(k)^{-1}$ is unitary, it follows that, for each $k \in \mathbb{R}^{d *}$, the set $\left\{u_{m}(k, s), v_{m}(k, s) \mid s=1, \ldots, \nu / 2\right\}$ is a complete orthonormal basis of $\mathbb{C}^{\nu}$. Hence

$$
\begin{aligned}
& \left\langle u_{m}(k, s), u_{m}\left(k, s^{\prime}\right)\right\rangle_{\mathbb{C}^{\nu}}=\delta_{s s^{\prime}} \\
& \left\langle v_{m}(k, s), v_{m}\left(k, s^{\prime}\right)\right\rangle_{\mathbb{C}^{\nu}}=\delta_{s s^{\prime}}, \\
& \left\langle u_{m}(k, s), v_{m}\left(k, s^{\prime}\right)\right\rangle_{\mathbb{C}^{\nu}}=0, \quad s, s^{\prime}=1, \ldots, \frac{\nu}{2} .
\end{aligned}
$$


and

$$
\sum_{s=1}^{\nu / 2}\left(u_{m r}(k, s) u_{m r^{\prime}}(k, s)^{*}+v_{m r}(k, s) v_{m r^{\prime}}(k)^{*}\right)=\delta_{r r^{\prime}}, \quad r, r^{\prime}=1, \ldots, \nu,
$$

where $u_{m r}(k, s)$ (resp. $v_{m r}(k, s)$ ) is the $r$ th component of the vector $u_{m}(k, s)$ (resp. $\left.v_{m}(k, s)\right)$ and, for a complex number $z \in \mathbb{C}, z^{*}$ denotes the complex conjugate of $z$.

The Hilbert space $\hat{\mathscr{H}}_{\mathrm{D}}$ has the orthogonal decomposition

$$
\hat{\mathscr{H}}_{\mathrm{D}}=\hat{\mathscr{H}}_{\mathrm{D}+} \oplus \hat{\mathscr{H}}_{\mathrm{D}-}
$$

with

$$
\hat{\mathscr{H}}_{\mathrm{D} \pm}:=L^{2}\left(\mathbb{R}^{d *} ; \mathbb{C}^{\nu / 2}\right) .
$$

We define linear operators $T_{m \pm}: \hat{\mathscr{H}}_{\mathrm{D}} \rightarrow \hat{\mathscr{H}}_{\mathrm{D} \pm}$ by

$$
\begin{aligned}
& T_{m+} f:=\left(u_{m}(\cdot, s)^{*} f\right)_{s=1}^{\nu / 2} \in \hat{\mathscr{H}}_{\mathrm{D}+}, \\
& T_{m-} f:=\left(\tilde{v}_{m}(\cdot, s) \tilde{f}\right)_{s=1}^{\nu / 2} \in \hat{\mathscr{H}}_{\mathrm{D}-}, \quad f \in \hat{\mathscr{H}}_{\mathrm{D}},
\end{aligned}
$$

where, for $w=\left(w_{r}\right)_{r=1}^{\nu}: \mathbb{R}^{d *} \rightarrow \mathbb{C}^{\nu}$,

$$
(w f)(k):=\sum_{r=1}^{\nu} w_{r}(k) f_{r}(k), \quad f=\left(f_{r}\right)_{r=1}^{\nu} \in \hat{\mathscr{H}}_{\mathrm{D}}, k \in \mathbb{R}^{d *}
$$

and

$$
\tilde{w}(k):=w(-k), \quad k \in \mathbb{R}^{d *} .
$$

It follows that $T_{m \pm}$ are bounded with $\left\|T_{m \pm}\right\| \leq \sqrt{\frac{\nu}{2}}$.

It is easy to see that

$$
\begin{aligned}
\left(T_{m+}^{*} f_{+}\right)_{r} & =\sum_{s=1}^{\nu / 2} u_{m r}(\cdot, s) f_{+s}, \quad f_{+}=\left(f_{+s}\right)_{s=1}^{\nu / 2} \in \hat{\mathscr{H}}_{\mathrm{D}+}, \\
\left(T_{m-}^{*} f_{-}\right)_{r} & =\sum_{s=1}^{\nu / 2} v_{m r}(\cdot, s)^{*} \tilde{f}_{-s}, \quad f_{-}=\left(f_{-s}\right)_{s=1}^{\nu / 2} \in \hat{\mathscr{H}}_{\mathrm{D}-}, r=1, \ldots, \nu .
\end{aligned}
$$

We denote by $C$ the complex conjugation on $\hat{\mathscr{H}}_{\mathrm{D}}$ :

$$
C f:=\left(f_{r}^{*}\right)_{r=1}^{\nu} .
$$

For a linear operator $A$ on $\hat{\mathscr{H}}_{\mathrm{D}}$, we define $\bar{A}$ by

$$
\bar{A}:=C A C .
$$

We regard $\hat{\mathscr{H}}_{\mathrm{D} \pm}$ as subspaces of $\hat{\mathscr{H}}_{\mathrm{D}}$ in the natural way so that $C$ acts also on $\hat{\mathscr{H}}_{\mathrm{D} \pm}$.

Remarkable properties of $T_{m \pm}$ are summarized in the following lemma: 


\section{Lemma 3.1}

$$
\begin{aligned}
& T_{m+}^{*} T_{m+}+\bar{T}_{m-}^{*} \bar{T}_{m-}=I, \\
& T_{m \pm} T_{m \pm}^{*}=I, \\
& T_{m+} \bar{T}_{m-}^{*}=0, \quad T_{m-} \bar{T}_{m+}^{*}=0 .
\end{aligned}
$$

Proof. Throughout the proof, we write $T_{ \pm}$(resp. $\left.u(k, s), v(k, s)\right)$ for $T_{m \pm}$ (resp. $\left.u_{m}(k, s), v_{m}(k, s)\right)$. Let $f, g \in \hat{\mathscr{H}}_{\mathrm{D}}$. Then

$$
\begin{aligned}
& \left\langle T_{+} f, T_{+} g\right\rangle+\left\langle\bar{T}_{-} f, \bar{T}_{-} g\right\rangle \\
& =\sum_{s=1}^{\nu / 2} \int_{\mathbb{R}^{d}}\left\{\left(u(\cdot, s) f^{*}\right)(k)\left(u(\cdot, s)^{*} g\right)(k)+\left(v(\cdot, s) f^{*}\right)(k)\left(v(\cdot, s)^{*} g\right)(k)\right\} d k \\
& =\sum_{r, r^{\prime}=1}^{\nu} \int_{\mathbb{R}^{d}} f_{r}(k)^{*} g_{r^{\prime}}(k)\left(\sum_{s=1}^{\nu / 2}\left(u_{r}(k, s) u_{r^{\prime}}(k, s)^{*}+v_{r}(k, s) v_{r^{\prime}}(k, s)^{*}\right)\right) d k \\
& =\sum_{r=1}^{\nu} \int_{\mathbb{R}^{d}} f_{r}(k)^{*} g_{r}(k) d k,
\end{aligned}
$$

where we have used (34) to obtain the last equality. Hence

$$
\left\langle T_{+} f, T_{+} g\right\rangle+\left\langle T_{-} f, T_{-} g\right\rangle=\langle f, g\rangle .
$$

This implies (40).

We have by (38)

$$
\begin{aligned}
\left\langle T_{+}^{*} f, T_{+}^{*} g\right\rangle & =\sum_{r=1}^{\nu} \sum_{s, s^{\prime}=1}^{\nu / 2}\left\langle u_{r}(\cdot, s) f_{s}, u_{r}\left(\cdot, s^{\prime}\right) g_{s^{\prime}}\right\rangle \\
& =\int_{\mathbb{R}^{d *}}\left\langle u(k, s), u\left(k, s^{\prime}\right)\right\rangle f_{s}(k)^{*} g_{s^{\prime}}(k) d k \\
& =\langle f, g\rangle,
\end{aligned}
$$

where we have used (31). Hence $T_{+} T_{+}^{*}=I$. Similarly, using (39), one can show that $T_{-} T_{-}^{*}=I$. One can see that orthogonality (33) implies (42).

Lemma 3.1 immediately yields the following result:

Lemma 3.2 For all $m \geq 0$,

$$
T_{m}:=\left(T_{m+}, T_{m-}\right)
$$

is an element of $\mathfrak{T}_{*}\left(\hat{\mathscr{H}}_{\mathrm{D}}\right)$.

It follows from (41) that $\left\|T_{m \pm}^{*}\right\|=1$ and hence

$$
\left\|T_{m \pm}\right\|=1 \text {. }
$$




\subsection{A free quantum Dirac field}

We construct a free quantum Dirac field on the fermion Fock space $\mathfrak{F}\left(\hat{\mathscr{H}}_{\mathrm{D}}\right)$ over $\hat{\mathscr{H}}_{\mathrm{D}}$. Note that we work with momentum representation. We denote by $a(f)\left(f \in \hat{\mathscr{H}}_{\mathrm{D}}\right)$ the annihilation operator on $\mathfrak{F}\left(\hat{\mathscr{H}}_{\mathrm{D}}\right)$.

For each $f \in \hat{\mathscr{H}}_{\mathrm{D}}$, we define

$$
\hat{\psi}_{m}(f):=a\left(T_{m+} f, 0\right)+a\left(0, T_{m-} f^{*}\right)^{*}, \quad f \in \hat{\mathscr{H}}_{\mathrm{D}}
$$

and set

$$
\left.\hat{\rho}_{m}:=\left\{\hat{\psi}_{m}(f), \hat{\psi}_{m}(f)^{*} \mid f \in \hat{\mathscr{H}}_{\mathrm{D}}\right\}\right) .
$$

Remark 3.3 A free quantum Dirac field on the $(1+d)$ (resp. 4)-dimensional space-time was considered in [5] (resp. [7]). In these papers (also in [8, §10.1]), the projection method as mentioned in Remark 2.4 is used. But we find that the projection method is somewhat inconvenient to discuss a family of quantum Dirac fields indexed by mass $\mathrm{m}$, since the orthogonal decomposition of $\mathscr{H}_{D}$ in the projection method depends on $\mathrm{m}$. Thus we take a slightly different approach in which the orthogonal decomposition (35) is fixed independently of $m$.

By Lemma 3.2 and an application of Theorem 2.7, we obtain the following proposition:

Proposition 3.4 For each $m \geq 0,\left(\mathfrak{F}\left(\hat{\mathscr{H}}_{\mathrm{D}}\right), \hat{\rho}_{m}\right)$ is an irreducible representation of the CAR over $\hat{\mathscr{H}}_{\mathrm{D}}$.

Let

$$
\psi_{m}(t, f):=\hat{\psi}_{m}\left(e^{i t \hat{H}_{m}} \hat{f}\right), \quad t \in \mathbb{R}, f \in D\left(H_{m}\right),
$$

and

$$
\rho_{m}(t):=\left\{\psi_{m}(t, f), \psi_{m}(t, f)^{*} \mid f \in \mathscr{H}_{\mathrm{D}}\right\}
$$

Since $e^{i t \hat{H}_{m}}$ is unitary for all $t \in \mathbb{R}$, Proposition 3.4 yields the following result:

Proposition 3.5 For each $m \geq 0$ and $t \in \mathbb{R},\left(\mathfrak{F}\left(\hat{\mathscr{H}}_{\mathrm{D}}\right), \rho_{m}(t)\right)$ is an irreducible representation of the CAR over $\mathscr{H}_{\mathrm{D}}$.

The following proposition shows that $\psi_{m}(t, f)$ is a free quantum Dirac field on the $(1+d)$-dimensional space-time and hence $\psi_{m}(\hat{f})=\psi_{m}(0, f)$ is the time-zero field of it.

Proposition 3.6 Let $m \geq 0$ and $f \in D\left(H_{m}\right)$. Then the operator-valued function: $t \mapsto$ $\psi_{m}(t, f) \in \mathfrak{F}\left(\hat{\mathscr{H}}_{\mathrm{D}}\right)$ on $\mathbb{R}$ is differentiable in the operator norm topology and obeys the free functional Dirac equation

$$
i \frac{d \psi_{m}(t, f)}{d t}=\psi_{m}\left(t, H_{m} f\right)
$$


Proof. Let $\varepsilon \in \mathbb{R} \backslash\{0\}$ and

$$
\phi_{\varepsilon}:=\frac{\psi_{m}(t+\varepsilon, f)-\psi_{m}(t, f)}{\varepsilon}-\psi_{m}\left(t, H_{m} f\right) .
$$

Then we have

$$
\phi_{\varepsilon}=\hat{\psi}_{m}\left(e^{i t \hat{H}_{m}} g_{\varepsilon}\right)
$$

where

$$
g_{\varepsilon}:=\left(-i \frac{\left(e^{i \varepsilon \hat{H}_{m}}-1\right)}{\varepsilon}-\hat{H}_{m}\right) \hat{f} .
$$

We have for all $h \in \hat{\mathscr{H}}_{\mathrm{D}}$

$$
\left\|\hat{\psi}_{m}(h)\right\| \leq\left\|T_{m+} h\right\|+\left\|T_{m-} h^{*}\right\| \leq 2\|h\|,
$$

where we have used (43). Hence $\left\|\phi_{\varepsilon}\right\| \leq 2\left\|g_{\varepsilon}\right\|$. Since $\hat{f} \in D\left(\hat{H}_{m}\right)$, it follows that $\lim _{\varepsilon \rightarrow 0}\left\|g_{\varepsilon}\right\|=0$. Hence $\lim _{\varepsilon \rightarrow 0}\left\|\phi_{\varepsilon}\right\|=0$.

One can show that, for all $f \in \mathscr{H}_{\mathrm{D}}$,

$$
\psi_{0, m}(t, f)=e^{i t \mathbb{H}_{0, m}} \hat{\psi}_{m}(\hat{f}) e^{-i t \mathbb{H}_{0, m}}, \quad t \in \mathbb{R},
$$

where $\mathbb{H}_{0, m}:=d \Gamma\left(E_{m}\right)$ is the second quantization of the multiplication operator $E_{m}$ (e.g., $\left[3\right.$, p.8]). The operator $\mathbb{H}_{0, m}$ is the Hamiltonian of the free quantum Dirac field with mass $m$ in momentum representation.

We now state and prove one of the main results in this paper:

Theorem 3.7 Let $m_{1} \neq m_{2}\left(m_{1}, m_{2} \geq 0\right)$. Then $\left(\mathfrak{F}\left(\hat{\mathscr{H}}_{\mathrm{D}}\right), \rho_{m_{1}}\right)$ and $\left(\mathfrak{F}\left(\hat{\mathscr{H}}_{\mathrm{D}}\right), \rho_{m_{2}}\right)$ are inequivalent.

Proof. By Theorem 2.11, we need only to prove that, if $m_{1} \neq m_{2}$, then $T_{m_{1}+} \bar{T}_{m_{2}-}^{*}$ or $T_{m_{1}-} \bar{T}_{m_{2}+}^{*}$ is not Hilbert-Schmidt. It is easy to see that

$$
\left(T_{m_{1}+} \bar{T}_{m_{2}-}^{*} f\right)_{r}(k)=\sum_{s=1}^{\nu / 2} K_{r s}(k) \tilde{f}_{s}(k), \quad f=\left(f_{s}\right)_{s=1}^{\nu / 2} \in \hat{\mathscr{H}}_{\mathrm{D}-}, r=1, \ldots, \frac{\nu}{2},
$$

where

$$
K_{r s}(k):=\left\langle u_{m_{1}}(k, r), v_{m_{2}}(k, s)\right\rangle_{\mathbb{C}^{\nu}}, \quad k \in \mathbb{R}^{d *}, r, s=1, \ldots, \frac{\nu}{2} .
$$

By (30), we have $K_{r s}(k)=\left\langle e_{r}, d_{m_{1}}(k) d_{m_{2}}(k)^{*} e_{s+\nu / 2}\right\rangle$. Using (26), (27), (21), (25) and the orthogonality $\left\langle e_{r}, e_{s+\nu / 2}\right\rangle=0(r, s=1, \ldots, \nu / 2)$, we obtain

$$
K_{r s}(k)=\left(m_{2}-m_{1}\right) F_{m_{1}, m_{2}}(k)\left\langle e_{r}, \alpha k e_{s+\nu / 2}\right\rangle, \quad r, s=1, \ldots, \frac{\nu}{2},
$$


where

$$
\begin{aligned}
F_{m_{1}, m_{2}}(k):= & \frac{1}{2 \sqrt{E_{m_{1}}(k) E_{m_{2}}(k)\left(m_{1}+E_{m_{1}}(k)\right)\left(m_{2}+E_{m_{2}}(k)\right)}} \\
& \times\left(1+\frac{m_{1}+m_{2}}{E_{m_{1}}(k)+E_{m_{2}}(k)}\right) .
\end{aligned}
$$

It follows from (6) that $\alpha k e_{s+\nu / 2} \in \operatorname{ker}(\beta-1), s=1, \ldots, \nu / 2$. Hence

$$
\sum_{r=1}^{\nu / 2}\left|\left\langle e_{r}, \alpha k e_{s+\nu / 2}\right\rangle\right|^{2}=\left\|\alpha k e_{s+\nu / 2}\right\|^{2}=k^{2},
$$

where we have used (25). Therefore

$$
\sum_{r=1}^{\nu / 2}\left|K_{r s}(k)\right|^{2}=\left(m_{2}-m_{1}\right)^{2} F_{m_{1}, m_{2}}(k)^{2} k^{2} .
$$

Let $m_{1} \neq m_{2}$. Suppose that $T_{m_{1}+} \bar{T}_{m_{2}-}^{*}$ were Hilbert-Schmidt. Denote by $\hat{K}_{r s}$ the multiplication operator by the function $K_{r s}(k)$ on $L^{2}\left(\mathbb{R}^{d *}\right)$. Then, by (48), $\hat{K}_{r s}$ is HilbertSchmidt. Hence $L:=\sum_{r=1}^{\nu / 2} \hat{K}_{r s}^{*} \hat{K}_{r s}$ is Hilbert-Schmidt. By (52), $L$ is the multiplication operator by the function $\left(m_{2}-m_{1}\right)^{2} F_{m_{1}, m_{2}}(k)^{2} k^{2}$. This function is continuous on $\mathbb{R}^{d *}$ and positive for all $|k|>0$. Hene $\sigma(L)$ includes an open interval in $[0, \infty)$. Hence $L$ is not Hilbert-Schmidt, since the spectrum of a Hilbert-Schmidt operator is purely discrete in $\mathbb{C} \backslash\{0\}$. Therefore we have a contradiction. Thus $T_{m_{1}+} \bar{T}_{m_{2}-}^{*}$ is not Hilbert-Schmidt.

\subsection{An interacting quantum Dirac field}

Let $\mathbb{H}_{m}$ be a self-adjoint operator on $\mathfrak{F}\left(\hat{\mathscr{H}}_{\mathrm{D}}\right)$ which may depend on $m$. Then the time- $t$ quantum Dirac field of mass $m$ with Hamiltonian $\mathbb{H}_{m}$ is defined by

$$
\psi_{m}(t, f):=e^{i t \mathbb{H}_{m}} \hat{\psi}_{m}(\hat{f}) e^{-i t \mathbb{H}_{m}}, \quad f \in \mathscr{H}_{\mathrm{D}}, t \in \mathbb{R} .
$$

This is a general form of interacting quantum Dirac fields whose time-zero field is taken to be $\hat{\psi}_{m}(\hat{f})$. The time- $t$ free quantum Dirac field $\psi_{0, m}(t, f)$ is given by the case where $\mathbb{H}_{m}=\mathbb{H}_{0, m}($ see $(47))$.

Let

$$
\rho_{m}(t):=\left\{\psi_{m}(t, f), \psi(t, f)^{*} \mid f \in \mathscr{H}_{\mathrm{D}}\right\}
$$

Then $\left(\mathfrak{F}\left(\hat{\mathscr{H}}_{\mathrm{D}}\right), \rho_{m}(t)\right)$ is an irreducible representation of the CAR over $\mathscr{H}_{\mathrm{D}}$. Since $e^{i t \mathbb{H}_{m}}$ is unitary, the following corollary immediately follows from Theorem 3.7:

Corollary 3.8 Let $m_{1} \neq m_{2}$. Then, for all $t \in \mathbb{R},\left(\mathfrak{F}\left(\hat{\mathscr{H}}_{\mathrm{D}}\right), \rho_{m_{1}}(t)\right)$ and $\left(\mathfrak{F}\left(\hat{\mathscr{H}}_{\mathrm{D}}\right), \rho_{m_{2}}(t)\right)$ are inequivalent. 


\section{A generalization}

In this section we briefly describe a generalization of the time-zero quantum Dirac field $\hat{\psi}(\hat{f})\left(f \in \mathscr{H}_{\mathrm{D}}\right)$.

Let $\mathscr{U}$ be the set of pairs $(u, v)$ of $\mathbb{C}^{\nu}$-valued Borel measurable functions $u$ and $v$ on $\mathbb{R}^{d *} \times\{1, \ldots, \nu / 2\}$ such that, for a.e. $k \in \mathbb{R}^{d *},\left\{u(k, s), v(k, s) \mid s=1, \ldots, \frac{\nu}{2}\right\}$ is an orthonormal basis of $\mathbb{C}^{\nu}$. For each $(u, v) \in \mathscr{U}$, we define $T_{+}(u): \hat{\mathscr{H}}_{\mathrm{D}} \rightarrow \hat{\mathscr{H}}_{\mathrm{D}+}$ and $T_{-}(v): \hat{\mathscr{H}}_{\mathrm{D}} \rightarrow \hat{\mathscr{H}}_{\mathrm{D}-}$ as follows (cf. (36) and (37)):

$$
\begin{aligned}
& T_{+}(u) f:=\left(u(\cdot, s)^{*} f\right)_{s=1}^{\nu / 2} \in \hat{\mathscr{H}}_{\mathrm{D}+}, \\
& T_{-}(v) f:=(\tilde{v}(\cdot, s) \tilde{f})_{s=1}^{\nu / 2} \in \hat{\mathscr{H}}_{\mathrm{D}-}, \quad f \in \hat{\mathscr{H}}_{\mathrm{D}} .
\end{aligned}
$$

Then, in the same way as in the proof of Lemma 3.1, one can prove the following relations:

$$
\begin{aligned}
& T_{+}(u)^{*} T_{+}(u)+\bar{T}_{-}(v)^{*} \bar{T}_{-}(v)=I, \\
& T_{+}(u) T_{+}(u)^{*}=I, \quad T_{-}(v) T_{-}(v)^{*}=I \\
& T_{+}(u) \bar{T}_{-}(v)
\end{aligned}
$$

Hence $\left(T_{+}(u), T_{-}(v)\right)$ is an element of $\mathfrak{T}_{*}\left(\hat{\mathscr{H}}_{\mathrm{D}}\right)$. Therefore, introducing the operators

$$
\hat{\psi}_{u, v}(f):=a\left(T_{+}(u) f, 0\right)+a\left(0, T_{-}(v) f^{*}\right)^{*}, \quad f \in \hat{\mathscr{H}}_{\mathrm{D}}
$$

and

$$
\hat{\rho}(u, v):=\left\{\hat{\psi}_{u, v}(f), \hat{\psi}_{u, v}(f)^{*} \mid f \in \hat{\mathscr{H}}_{\mathrm{D}}\right\},
$$

we see that $\left(\mathfrak{F}\left(\hat{\mathscr{H}}_{\mathrm{D}}\right), \hat{\rho}(u, v)\right)$ is an irreducible representation of the CAR over $\hat{\mathscr{H}}_{\mathrm{D}}$. Clearly this class of representations includes $\left(\mathfrak{F}\left(\hat{\mathscr{H}}_{\mathrm{D}}\right), \hat{\rho}_{m}\right)$, hence being a generalization of it.

As for the family $\left\{\left(\mathfrak{F}\left(\hat{\mathscr{H}}_{\mathrm{D}}\right), \hat{\rho}(u, v)\right) \mid(u, v) \in \mathscr{U}\right\}$ of representations of the CAR over $\hat{\mathscr{H}}_{\mathrm{D}}$, we have the following inequivalence theorem:

Theorem 4.1 Let $\left(u_{1}, v_{1}\right)$ and $\left(u_{2}, v_{2}\right)$ be in $\mathscr{U}$. Then the two representations $\left(\mathfrak{F}\left(\hat{\mathscr{H}}_{\mathrm{D}}\right), \hat{\rho}\left(u_{1}, v_{1}\right)\right)$ and $\left(\mathfrak{F}\left(\hat{\mathscr{H}}_{\mathrm{D}}\right), \hat{\rho}\left(u_{2}, v_{2}\right)\right)$ are inequivalent if and only if, for some $(r, s)$, there exists a non-null Borel set $B \subset \mathbb{R}^{d *}$ such that, for all $k \in B,\left\langle u_{1}(k, r), v_{2}(k, s)\right\rangle_{\mathbb{C}^{\nu}} \neq 0$ or $\left\langle u_{2}(k, r), v_{1}(k, s)\right\rangle_{\mathbb{C}^{\nu}} \neq 0$.

Proof. By Theorem 2.11, the two representations under consideration are inequivalent if and only if $T_{+}\left(u_{1}\right){\overline{T_{-}\left(v_{2}\right)}}^{*}$ or $T_{-}\left(v_{1}\right){\overline{T_{+}\left(u_{2}\right)}}^{*}$ is not Hilbert-Schmidt.

In the same way as in the case of (48), one can show that

$$
\left(T_{+}\left(u_{1}\right){\overline{T_{-}\left(v_{2}\right)}}^{*} f\right)_{r}(k)=\sum_{s=1}^{\nu / 2} G_{r s}(k) f_{s}(k), \quad f \in \hat{\mathscr{H}}_{\mathrm{D}}, r=1, \ldots, \frac{\nu}{2}, \text { a.e. } k,
$$


where

$$
G_{r s}(k):=\left\langle u_{1}(k, r), v_{2}(k, s)\right\rangle_{\mathbb{C}^{\nu}} .
$$

It is easy to see that $T_{+}\left(u_{1}\right){\overline{T_{-}\left(v_{2}\right)}}^{*}$ is not Hilbert-Schmidt if and only if, for some $(r, s)$, the multiplication operator $\hat{G}_{r s}$ by the function $G_{r s}$ is not Hilbert-Schmidt. In general, the multiplication operator on $L^{2}\left(\mathbb{R}^{d *}\right)$ by a function $F$ is Hilbert-Schmidt if and only if $F(k)=0$ a.e. $k \in \mathbb{R}^{d *}$. Hence $\hat{G}_{r s}$ is not Hilbert-Schmidt if and only if there exists a non-null Borel set $B \subset \mathbb{R}^{d *}$ such that, for all $k \in B, G_{r s}(k) \neq 0$. Thus $T_{+}\left(u_{1}\right) \bar{T}_{-}\left(v_{2}\right) *$ is not Hilbert-Schmidt if and only if, for some $(r, s)$, there exists a non-null Borel set $B \subset \mathbb{R}^{d *}$ such that, for all $k \in B, G_{r s}(k) \neq 0$.

Note that $T_{-}\left(v_{1}\right){\overline{T_{+}\left(u_{2}\right)}}^{*}$ is not Hilbert-Schmidt if and only if $T_{+}\left(u_{2}\right){\overline{T_{-}\left(v_{1}\right)}}^{*}$ is not Hilbert-Schmidt. Hence one can apply the preceding result to the case $\left(u_{1}, v_{2}\right)$ replaced by $\left(u_{2}, v_{1}\right)$ to conclude that $T_{-}\left(v_{1}\right){\overline{T_{+}\left(u_{2}\right)}}^{*}$ is not Hilbert-Schmidt if and only if, for some $(r, s)$, there exists a non-null Borel set $B \subset \mathbb{R}^{d *}$ such that, $\left\langle u_{2}(k, r), v_{1}(k, s)\right\rangle_{\mathbb{C}^{\nu}} \neq 0$.

\section{The case of quantum Dirac fields on $d$-dimensional boxes}

In [2, Theorem 6.12], it is shown that the time-zero quantum scalar fields of different positive masses on a bounded region in $\mathbb{R}^{d}$ are equivalent if and only if $d \leq 3$, in contrast to the case of the infinite space $\mathbb{R}^{d}$. It is natural to ask if quantum Dirac fields on a bounded region in $\mathbb{R}^{d}$ have similar properties. In this section we give an answer to this question. For simplicity, we consider quantum Dirac fields on the $d$-dimensional box

$$
M:=\mathbb{I}_{1} \times \cdots \times \mathbb{I}_{d}, \quad \mathbb{I}_{j}:=\left[-\frac{L_{j}}{2}, \frac{L_{j}}{2}\right]\left(L_{j}>0, j=1, \ldots, d\right) .
$$

Let

$$
\Gamma_{j}:=\left\{\frac{2 \pi}{L_{j}} n \mid n \in \mathbb{Z}\right\},
$$

where $\mathbb{Z}$ is the set of all integers, and

$$
\Gamma:=\Gamma_{1} \times \cdots \times \Gamma_{d}=\left\{k=\left(k_{1}, \ldots, k_{d}\right) \mid k_{j} \in \Gamma_{j}, j=1, \ldots, d\right\} .
$$

For each $k \in \Gamma$, we define a function $\phi_{k}$ on $M$ by

$$
\phi_{k}(x):=\frac{1}{\sqrt{L_{1} \cdots L_{d}}} e^{i k x}, \quad x \in M .
$$

It is well known that $\left\{\phi_{k} \mid k \in \Gamma\right\}$ is a complete orthonormal system (CONS) of $L^{2}(M)$. Hence the mapping $U_{d}: L^{2}(M) \rightarrow \ell^{2}(\Gamma)$ (the Hilbert space of absolutely square summable sequences on $\Gamma$ ) defined by

$$
U_{d} f(k):=\left\langle\phi_{k}, f\right\rangle_{L^{2}(M)}, \quad f \in L^{2}(M), k \in \Gamma,
$$


is unitary.

For each $j=1, \ldots, d$, the multiplication operator by the $j$ th coordinate function $k_{j}$ in $\Gamma$ is self-adjoint. We denote it by the same symbol $k_{j}$. Then the operator

$$
p_{j}^{(M)}:=U_{d}^{-1} k_{j} U_{d}
$$

is a self-adjoint operator on $L^{2}(M)$ with $D\left(p_{j}^{(M)}\right)=U_{d}^{-1} D\left(k_{j}\right)$. The operator $p_{j}^{(M)}$ is called the $j$ th momentum operator in $M$ with the periodic boundary condition.

As a free Dirac operator on the Hilbert space

$$
\mathscr{H}_{\mathrm{D}}^{(M)}:=L^{2}\left(M ; \mathbb{C}^{\nu}\right)
$$

with mass $m>0$, we take the following one:

$$
H_{m}^{(M)}:=\sum_{j=1}^{d} \alpha_{j} p_{j}^{(M)}+m \beta .
$$

The operator $H_{m}^{(M)}$ is self-adjoint and the operator equality

$$
U_{d} H_{m}^{(M)} U_{d}^{-1}=h_{m}^{(M)}
$$

holds with $h_{m}^{(M)}$ being the multiplication operator on the Hilbert space

$$
\hat{\mathscr{H}}_{\mathrm{D}}^{(M)}:=U_{d} \mathscr{H}_{\mathrm{D}}^{(M)}=\oplus^{\nu} \ell^{2}(\Gamma) .
$$

by the matrix-valued function

$$
h_{m}^{(M)}(k):=\sum_{j=1}^{d} \alpha_{j} k_{j}+m \beta, \quad k \in \Gamma .
$$

As in the case of the infinite space $\mathbb{R}^{d}$, we have

$$
\hat{\mathscr{H}}_{\mathrm{D}}^{(M)}=\hat{\mathscr{H}}_{\mathrm{D}+}^{(M)} \oplus \hat{\mathscr{H}}_{\mathrm{D}-}^{(M)},
$$

where $\hat{\mathscr{H}}_{\mathrm{D} \pm}^{(M)}:=\oplus^{\nu / 2} \ell^{2}(\Gamma)$.

Objects in Section 3 such as $E_{m}, d_{m}, u_{m}, v_{m}$ have counterparts in the theory on $M$ in an obvious way. We write them with upper suffix " $(M)$ " (e.g., $\left.E_{m}^{(M)}(k):=E_{m}(k), k \in \Gamma\right)$. We define the operators $T_{m \pm}^{(M)}: \hat{\mathscr{H}}_{\mathrm{D}}^{(M)} \rightarrow \hat{\mathscr{H}}_{\mathrm{D} \pm}^{(M)}$ by $T_{m \pm}$ with $u_{m}$ and $v_{m}$ replaced by $u_{m}^{(M)}$ and $v_{m}^{(M)}$ respectively. Then it is easy to see that Lemma 3.1 holds with $T_{m \pm}$ replaced by $T_{m \pm}^{(M)}$.

We denote by $a_{M}(\cdot)$ the annihilation operator on the fermion Fock space $\mathfrak{F}\left(\hat{\mathscr{H}}_{\mathrm{D}}^{(M)}\right)$ and define a quantum Dirac field on $M$ by

$$
\psi_{m}^{(M)}(f):=a_{M}\left(T_{m+}^{(M)} U_{d} f, 0\right)+a_{M}\left(0, T_{m-}^{(M)}\left(U_{d} f\right)^{*}\right)^{*}, \quad f \in \mathscr{H}_{\mathrm{D}}^{(M)} .
$$


Let

$$
\rho_{m}^{(M)}:=\left\{\psi_{m}^{(M)}(f), \psi_{m}^{(M)}(f)^{*} \mid f \in \mathscr{H}_{\mathrm{D}}^{(M)}\right\} .
$$

Then, as in Proposition 3.4, we can show that $\left(\mathfrak{F}\left(\hat{\mathscr{H}}_{\mathrm{D}}^{(M)}\right), \rho_{m}^{(M)}\right)$ is an irreducible representation of the CAR over $\mathscr{H}_{\mathrm{D}}^{(M)}$. Now we are ready to prove the following theorem:

Theorem 5.1 Let $m_{1} \neq m_{2}\left(m_{1}, m_{2}>0\right)$. Then $\left(\mathfrak{F}\left(\hat{\mathscr{H}}_{\mathrm{D}}^{(M)}\right), \rho_{m_{1}}^{(M)}\right)$ is equivalent to $\left(\mathfrak{F}\left(\hat{\mathscr{H}}_{\mathrm{D}}^{(M)}\right), \rho_{m_{2}}^{(M)}\right)$ if and only if $d=1$.

Proof. Throughout the proof, we write $T_{j \pm}:=T_{m_{j} \pm}^{(M)}, j=1,2$. It is easy to see that

$$
\begin{aligned}
\left(T_{1+} \bar{T}_{2-}^{*} w\right)_{r}(k)= & \sum_{s=1}^{\nu / 2} K_{r s}(k) \tilde{w}_{s}(k), \\
& w=\left(w_{s}\right)_{s=1}^{\nu / 2} \in \hat{\mathscr{H}}_{\mathrm{D}-}^{(M)}, r=1, \ldots, \frac{\nu}{2}, k \in \Gamma,
\end{aligned}
$$

where $K_{r s}$ is defined by (49) with domain $\Gamma$. For each $k \in \Gamma$, we define a vector $e_{k} \in \ell^{2}(\Gamma)$ by

$$
e_{k}\left(k^{\prime}\right)=\delta_{k k^{\prime}}, \quad k^{\prime} \in \Gamma .
$$

It is easy to see that $\left\{e_{k}\right\}_{k \in \Gamma}$ is a CONS of $\ell^{2}(\Gamma)$. For $s=1, \ldots, \nu / 2$, we define

$$
e_{k}^{(s)}=\left(0, \ldots, 0, e_{k}^{s t h}, 0, \ldots, 0\right) \in \hat{\mathscr{H}}_{\mathrm{D}-}^{(M)} .
$$

Then $\left\{e_{k}^{(s)} \mid k \in \Gamma, s=1, \ldots, \nu / 2\right\}$ is a CONS of $\hat{\mathscr{H}}_{\mathrm{D}-}^{(M)}$. We have

$$
\left(T_{1+} \bar{T}_{2-}^{*} e_{k}^{(s)}\right)_{r}\left(k^{\prime}\right)=K_{r s}\left(k^{\prime}\right) \delta_{k,-k^{\prime}}, \quad k^{\prime}, k \in \Gamma .
$$

Hence

$$
\sum_{s=1}^{\nu / 2} \sum_{k \in \Gamma}\left\|T_{1+} \bar{T}_{2-}^{*} e_{k}^{(s)}\right\|^{2}=\sum_{r, s=1}^{\nu / 2} \sum_{k \in \Gamma}\left|K_{r s}(k)\right|^{2} .
$$

Hence $T_{1+} \bar{T}_{2-}^{*}$ is Hilbert-Schmidt if and only if $\sum_{k \in \Gamma}\left|K_{r s}(k)\right|^{2}<\infty$ for $r, s,=1, \ldots, \nu / 2$. In the present case too, we have (52). Hence it follows that $T_{1+} \bar{T}_{2-}^{*}$ is Hilbert-Schmidt if and only if $\sum_{k \in \Gamma} F_{m_{1}, m_{2}}(k)^{2} k^{2}<\infty$.

It is easy to see that

$$
\frac{1}{2\left(m_{1}+E_{m_{1}}(k)\right)\left(m_{2}+E_{m_{2}}(k)\right)} \leq F_{m_{1}, m_{2}}(k) \leq \frac{1}{E_{m_{1}}(k) E_{m_{2}}(k)}, \quad k \in \Gamma .
$$

Hence, for each $R>0$, there exist positive constants $C_{1}$ and $C_{2}$ such that, for all $|k| \geq R$,

$$
\frac{C_{1}}{k^{2}} \leq F_{m_{1}, m_{2}}(k) \leq \frac{C_{2}}{k^{2}}
$$


Therefore

$$
\frac{C_{1}^{2}}{k^{2}} \leq F_{m_{1}, m_{2}}(k)^{2} k^{2} \leq \frac{C_{2}^{2}}{k^{2}} .
$$

It is obvious that $\sum_{k \in \Gamma} 1 / k^{2}<\infty$ if and only if $d=1$. Hence $T_{1+} \bar{T}_{2-}^{*}$ is Hilbert-Schmidt if and only if $d=1$. Similarly, we see that $T_{1-} \bar{T}_{2+}^{*}$ is Hilbert-Schmidt if and only if $d=1$. Thus, by Theorem 2.10, we obtain the desired result.

Remark 5.2 Theorem 5.1 is interesting in tha it is different from a corresponding theorem in the theory of quantum scalar fields on $M$ [2, Theorem 6.12], where, in the cases $d=2,3$ too, the time-zero quantum scalar fields of different masses are equivalent.

\section{References}

[1] A. Arai, Fock Spaces and Quantum Fields (in Japanese). Nippon-hyouronsha, Tokyo, 2000.

[2] A. Arai, A family of inequivalent Weyl representations of canonical commutation relations with applications to quantum field theory. Hokkaido University Preprint Series in Mathematics \#1085, 2015. http://www.math.sci.hokudai.ac.jp/preprint/

[3] O. Bratteli and D. W. Robinson, Operator Algebras and Quantum Statistical Mechanics 2. Second Edition, Springer-Verlag, Berlin Heidelberg, 1997.

[4] J. T. Ottesen, Infinite Dimensional Groups and Algebras in Quantum Physics. Springer-Verlag, Berlin Heidelberg, 1995.

[5] J. Palmer, Scattering automorphisms of the Dirac field. J. Math. Anal. Appl. 64 1978), 189-215.

[6] M. Reed and B. Simon, Methods of Modern Mathematical Physics II: Fourier Analysis, Self-Adjointness. Academic Press, New York, 1975.

[7] S. N. M. Ruijsenaars, Charged particles in external fields. II. The quantized Dirac and Klein-Gordon theories. Commun. Math. Phys. 52 (1977), 267-294.

[8] B. Thaller, The Dirac Equation. Springer-Verlag, Berlin Heidelberg, 1992. 